\title{
The design of a new truncated and engineered alpha1-antitrypsin based on theoretical studies: an antiprotease therapeutics for pulmonary diseases
}

\author{
Nazanin Pirooznia', Sadegh Hasannia ${ }^{2,3^{*}}$, Seyed Shahriar Arab $^{4}$, Abbas Sahebghadam Lotfi, ${ }^{3,5}$, Mostafa Ghanei ${ }^{6}$ \\ and Abbas Shali ${ }^{3}$
}

\section{* Correspondence:}

hasannia@nigeb.ac.ir

²Department of Biochemistry,

School of Biological Sciences,

Tarbiat Modares University, Tehran,

Iran

${ }^{3}$ National Institute of Genetic

Engineering and Biotechnology,

Tehran, Iran

Full list of author information is

available at the end of the article

\section{( Biomed Central}

\begin{abstract}
Alpha 1- antitrypsin (a1AT) a $54 \mathrm{kDa}$ glycoprotein is a protease inhibitor. In the absence of a1AT, elastase released by lung macrophages, was not inhibited and lead to elastin breakdown and pulmonary problems such as emphysema or COPD. a1AT has three site of $\mathrm{N}$-glycosylation and a characteristic reactive central loop (RCL). As small-scale medicines are preferred for pulmonary drug delivery, in this study a1ATs $(1,2,3,4$ and 5$)$ were engineered and shortened from the N-terminal region. In order to investigate the effect of different mutations and the deletion of 46 amino acids theoretical studies were performed. Homology modeling was performed to generate the 3D structure of a1ATs. The 10 ns Molecular Dynamic (MD) simulations were carried out to refine the models. Results from MD and protein docking showed that a1AT2 has the highest binding affinity for neutrophil elastase, provided the basis for the experimental phase in which sequences from the five a1AT constructs were inserted into the expression vector pGAPZa and expressed in the yeast Pichia pastoris. Although, the a1AT2 construct has the highest inhibitory activity even more that the native construct (a1AT5), results indicated the presence of protease inhibitory function of all the proteins' construct against elastase.
\end{abstract}

Keywords: Alpha 1- antitrypsin, Molecular dynamics (MD) simulation, Neutrophil elastase, Protein docking

\section{Introduction}

a1AT is a $54 \mathrm{kDa}$ glycoprotein which is a serine protease and broad group of other protease inhibitor. This protein protect the lung from cellular inflammatory enzymes, especially neutrophil (leukocyte) elastase, therefore it is called human neutrophil elastase inhibitor [1-3]. In the absence of $\alpha 1 \mathrm{AT}$, elastase released from neutrophils, was not inhibited and lead to elastin breakdown and other symptoms like pulmonary problems such as emphysema or COPD in adults [2,3]. Impaired $\alpha 1 \mathrm{AT}$ secretion in liver and accumulation in liver cells also cause cirrhosis in neonatal [4]. Relation between $\alpha 1 \mathrm{AT}$ and numbers of diseases including asthma, rheumatoid arthritis, anterior uveitis and systemic lupus erythematosus suggest that $\alpha 1 \mathrm{AT}$ is not only an anti- inflammation protein but also an immune system regulator [5-8]. $\alpha 1 \mathrm{AT}$ regulates lymphocyte

(c) 2013 Pirooznia et al.; licensee BioMed Central Ltd. This is an Open Access article distributed under the terms of the Creative Commons Attribution License (http://creativecommons.org/licenses/by/2.0), which permits unrestricted use, distribution, and reproduction in any medium, provided the original work is properly cited. 
proliferation and cytotoxicity, mediates monocytes and neutrophils function. Also $\alpha 1 \mathrm{AT}$ is suggested as an antiapoptotic factor in lung epithelial cells [9]. Besides it was illustrated that protease-antiprotease imbalance is an important factor in COPD and other pulmonary diseases pathogenesis such as bronchitis. In this process exogenous proteolytic enzymes leads to lung breakdown (because human neutrophil elastase cannot be inhibited by $\alpha 1 \mathrm{AT}$ ) [10]. Cigarette smoking is considered as the main cause of COPD which is the fourth leading cause of death in the United States [2]. The oxidation of Met358 in the reactive center loop (RCL) of $\alpha 1 \mathrm{AT}$ leads to a critical decrease in inhibitory capacity of this protein against elastase which cause inactivation in protective function of this protein [11]. It was shown that oxidation sensitivity is a regulatory process, and $\alpha 1 \mathrm{AT}$ inactivation possibly causing lung tissue breakdown in inflammatory site through oxygen radicals released from phagocytes. Therefore, smoking causes oxidation of critical residues (methionine) in $\alpha 1 \mathrm{AT}$ and inactivates this protein. $\alpha 1$ AT resistant to oxidation can improve health conditions in COPD patients and also reduction of apoptosis induced by cigarette smoking in vitro. $\alpha 1 \mathrm{AT}$ beside antiapoptotic function in in vitro, has broad anti inflammatory effects in human. Therefore treatment of CF patients with aerosolized $\alpha 1 \mathrm{AT}$ has been shown to reduce sputum neutrophil numbers, IL-8 concentration and elastase function. The effect of $\alpha 1 \mathrm{AT}$ on IL-8 as a neutrophil-chemoattractant and neutrophil recruitment to lung is also important because neutrophils are considered as the main cause of COPD pathogenesis and clinical effects of $\alpha 1 \mathrm{AT}$ in COPD are obvious [7].

Nowadays the only intravenous infusion of $\alpha 1 \mathrm{AT}$ is available in the world as therapeutic agent which is not the first choice for COPD treatment due to its high cost and low availability [12]. But the aerosol form of $\alpha 1 \mathrm{AT}$ in CF and COPD patients for the prevention of disease progression is not hard to believe [13].

a1AT in its mature has a polypeptide chain consisting of one cystine, two tryptophans and nine methionine residues. $\alpha 1 \mathrm{AT}$ has three site of $\mathrm{N}$-glycosylation which are highly mannosylated because tri- and tetra $\mathrm{N}$-glycans can be attached to aspargine $107[1]$.

The oxidation of Met358 in the reactive center loop (RCL) of $\alpha 1 \mathrm{AT}$ leads to a critical decrease in inhibitory capacity of this protein against elastase which cause inactivation in protective function of this protein. It was shown that oxidation sensitivity is a regulatory process, and $\alpha 1 \mathrm{AT}$ inactivation possibly causing lung tissue breakdown in inflammatory site through oxygen radicals released from phagocytes [14,15].

Several mutations within the $\alpha 1 \mathrm{AT}$ genes have been found with the most common point mutations including S and Z mutations with Glu264Val and Glu342Lys substitutions, respectively [16]. FDA has confirmed the utilization of four human plasma derived products of $\alpha 1$ AT-Prolastin, Zemaira, Aralast and Glassia as replacement or augmentation therapy. These products which increase the intravenous concentration of $\alpha 1 \mathrm{AT}$, cost more than $100000 \$$ for each person annually. The infusion form of $\alpha 1 \mathrm{AT}$ has several disadvantages such as: because it has blood origin the risk of viral and non viral contamination is high, also due to the limitation of proteins extracted from blood, huge production is not possible $[10,12,17]$.

The aerosolized $\alpha 1 \mathrm{AT}$ are under study. In the aerosol form, $25 \%-45 \%$ of aerosolized particle reach the respiratory system but in intravenous infusion only $10 \%-15 \%$ of a1AT reach to this site. The aerosolized $\alpha 1 \mathrm{AT}$ not only affect locally its main site of 
action but also avoid remaining and circulation for a long time in peripheral blood and therefore depress immune response reactions because of long-term use of $\alpha 1 \mathrm{AT}$ [18].

In the other hand, yeasts are effective eukaryotic systems in recombinant protein synthesis in large scale and are able to perform post translational modifications such as glycosylation. In spite of the useful properties of yeasts, these cells are not effective enough for the secretion of high molecular weight glycoproteins [19-22]. Therefore any modification which possibly increases the efficacy and secretion of high molecular weight glycoproteins in yeast can have significant results in protein based medicine products in these cells. With the reduction in size of $\alpha 1 \mathrm{AT}$ beside the preservation of function in production process and increase in the secretion ability of yeast, alteration in glycosylation patterns and elimination of some carbohydrate chains reduce the immunogenicity of secreted $\alpha 1 \mathrm{AT}$, as a yeast characteristic property is addition of huge mannosylation patterns to secreted proteins.

Huge mannosylation pattern in yeast increases the molecular weight significantly, possibly affects protein activity and also causes difficulty and disturbance in drug deliver to the lung.

Therefore, charbohydrate chains deletion from recombinant $\alpha 1 \mathrm{AT}$ are in the majority of this project. Then, protein length from $\mathrm{N}$-terminal was reduced to produce a smaller molecule which has the same and even higher inhibitory activity as the native $\alpha 1 \mathrm{AT}$. So, a new $\alpha 1 \mathrm{AT}$ structures were obtained that can be appropriate candidates for pulmonary drug delivery. Therefore, five different $\alpha 1$ AT structures with different glycosylation patterns and molecular weights were constructed base of theoretical studies. Beside, each $\alpha 1 \mathrm{AT}$ structures were subjected to three or five different site directed mutagenesis at the same time for both thermal and oxidative inactivation stability. Inhibitory properties of each $\alpha 1 \mathrm{AT}$ constructs were investigated through Elastase Inhibitory Concentration (EIC). Previous studies have shown that the two methionine residues 351 and 358, and in sever conditions methionine 226 are susceptible to oxidation. Oxidation of methionine 358 leads the loss of anti-elastase activity of $\alpha 1 \mathrm{AT}$. The other methionine residue (Met351) is also as susceptible to oxidation and anti-elastase activity loss as Met358 [23,24].

In this study, site directed mutations in $\alpha 1 \mathrm{AT}$, produce an active, lower molecular weight, resistant to oxidation and thermal inactivation, with an appropriate half life and unglycosylated protein in Pichia Pastoris as a host. In this study we showed that mutations and engineering of the protein molecule, lead to an improvement in inhibitory function. Furthermore, a protein with a less molecular weight and immunogenicity which is suitable for drug packaging and targeting to the lung which is the site of a1AT's action was produce. Molecular dynamic studies showed that deletion of the first alpha-helix from $\mathrm{N}$-terminal sequence have no effect on $\alpha 1 \mathrm{AT}$ inhibitory function. Therefore a reduction of $7 \mathrm{kDa}$ in molecular weight of $\alpha 1 \mathrm{AT}, 5 \mathrm{kDa}$ contributing to amino acids weight and approximating $2 \mathrm{kDa}$ to the first carbohydrate chain, was resulted.

\section{Methods and materials} a1AT model preparation

Five different protein sequences of $\alpha 1 \mathrm{AT}$ namely $\alpha 1 \mathrm{AT}_{1}, \alpha 1 \mathrm{AT}_{2}, \alpha 1 \mathrm{AT}_{3}, \alpha 1 \mathrm{AT}_{4}$ and $\alpha 1 \mathrm{AT}_{5}$ were selected (Table 1). Homology modeling of $\alpha 1 \mathrm{AT}$ subtypes was performed using as 
Table 1 Engineered, truncated a1AT structures

\begin{tabular}{lcccc}
\hline Structure & $\begin{array}{c}\text { Number of } \\
\text { residue }\end{array}$ & $\begin{array}{c}\text { Molecular } \\
\text { weight }\end{array}$ & Mutation sites & $\begin{array}{c}\text { N-terminal } \\
\text { deletion }\end{array}$ \\
\hline a1AT1 & 350 & 39342.3 & M351V, M358L, M226L & 46 aa \\
a1AT2 & 396 & 44560.8 & M351V, M358L, M226L, N83Q, N243Q & - \\
a1AT3 & 350 & 39328.2 & M351V, M358L, M226L & 46 aa \\
a1AT4 & 396 & 44560.8 & M351V, M358L, M226L, N46Q, N243Q & - \\
a1AT5 & 396 & 44532.8 & M351V, M358L, M226L & - \\
\hline
\end{tabular}

template the X-ray structure at $2.0 \AA$ Resolution of the intact alpha 1-antitrypsin, PDB code file 1QLP. I-TASSER server was applied for the model construction. Structures with the lowest objective function were used for performing molecular dynamic simulation [25].

\section{Molecular dynamics simulations}

The MD simulations were carried out for 10 ns using GROMACS 4.5.3 software package with the standard GROMOS43a1 force field in the presence of explicit solvent molecules and $\mathrm{Na}$ counterions $[26,27]$.

The energy of these systems was minimized using the steepest descent approach realized in the GROMACS package to remove close Van der Waals contacts and to allow formation of hydrogen bonds between water molecules in the periodic box and the protein. A 100 ps MD simulations were performed at the canonical ensemble (NVT) and the temperature was increased from 200 to $300 \mathrm{~K}$. The simulations were continued for another 100 ps at the isobaric-isothermal ensemble (NPT) so that the systems reached equilibration at a constant pressure. The density was stabilized around $0.98 \mathrm{~g} / \mathrm{cm}^{3}$ during equilibration phase in constant pressure. Finally, a $10 \mathrm{~ns}$ MD simulations were performed at the NPT canonical ensemble and the periodic boundary conditions were used in all three dimensions. NVT ensemble simulation was carried out using TIP3P as water models. In all simulations, to keep all hydrogen bonds rigid the SHAKE algorithm was used. The mesh Ewald (PME) summation technique was used to calculate long-range electrostatics interactions; a $14 \mathrm{~A}^{\circ}$ cutoff for van der Walls interactions, a $12 \mathrm{~A}^{\circ}$ cutoff for Coulomb interaction with updates every 10 steps, where a time step of 2 fs was considered. In all simulations, the SHAKE algorithm was used to fix hydrogen bond trembling with other atoms. MD simulations were performed in water in a cubic box and $8 \mathrm{~A}^{\circ}$ water layer thickness was considered.

Analyses of RMSD, RMSF, solvent accessible surface area, gyration radius, secondary structure, number of hydrogen bonds and dihedral-angle structural distance maps have been carried out using the GROMACS 4.5.3 analysis tools. The first 5 ns of simulation time were discarded to take into account the long system equilibration and all the analyses have been performed on the last $5 \mathrm{~ns}$.

\section{Molecular docking}

The calculations regarding the interaction between neutrophil elastase and $\alpha 1 \mathrm{AT}$ structures, which were obtained by MD simulations, were performed using 3D-Dock program. The structure of neutrophil elastase was obtained from the Protein Data Bank (PDB ID: 2D26). Protein dockings were carried out using MultiDock software package. 
Docked structures were constructed using FTDock program. The surface thicknesses of $\alpha 1$ ATs were considered as $1.3 \AA$, and the RPScore program was used to score all known complexes. For each calculation, 100 complexes with the highest score were selected using the MultiDock program. Finally, the complexes at the lowest energy levels were selected and compared with each other.

\section{Bacteria and $P$. pastoris strains and media}

The E. coli strain $D H 5 \alpha$ was used for propagation of recombinant plasmids. The P. pastoris strain $X$-33 (Invitrogen) was used as a host for the protein expression. Recombinant bacteria were cultured in low salt LB broth medium $(0.5 \%(\mathrm{w} / \mathrm{v})$ yeast extract, $1 \%(\mathrm{w} / \mathrm{v})$ tryptone, $0.5 \%(\mathrm{w} / \mathrm{v}) \mathrm{NaCl}$ ) supplemented with $25 \mu \mathrm{g} \mathrm{ml-1}$ of zeocin (Invitrogen). P. pastoris was cultured on the following medium: YPDS plates (1\% (w/v) yeast extract, $2 \%(\mathrm{w} / \mathrm{v})$ peptone, $2 \%(\mathrm{w} / \mathrm{v})$ dextrose, $1 \mathrm{M}$ sorbitol, and $1.5 \%(\mathrm{w} / \mathrm{v})$ bacteriological agar) supplemented with $100 \mu \mathrm{g} / \mathrm{ml}$ of zeocin.

\section{Cloning of a1AT in pGAPZaA plasmid}

For the construction of the expression vector EcoRI and $X b a \mathrm{I}$ sites were introduced respectively at the $5^{\prime}$ and $3^{\prime}$ end of $\alpha 1$ ATs by PCR with specific primers (Table 2). Using DNA from the previous work as template, the resulting 1059 and 1197 bp PCR product was cloned into the pGAPZ $\alpha$ A plasmid (Invitrogen, USA).

\section{P. pastoris transformation and selection}

P. pastoris cells were grown overnight in YPD broth $\left(\right.$ at $\left.28^{\circ} \mathrm{C} / 250 \mathrm{rpm}\right)$ and prepared for transformation according to the manufacturer's recommendations (Invitrogen). The recombinant plasmid was linearized with BspHI in the GAP promoter region and electrotransformed into P. pastoris X33 electrocompetent cells using a Bio-Rad genepulser apparatus (at $1.5 \mathrm{kV}, 25 \mathrm{~F}, 400 \Omega$ and $8 \mathrm{~ms}$ ). Zeocin resistant transformants were selected on YPDS agar plates. P. pastoris X33 with empty vector was transformed for negative control tests. Integration analysis was confirmed by PCR following with the primers $p G A P$ forward and 3 'AOX1 reverse. Correct integration will result in the formation of $\sim 1050-1200$ bp PCR products.

\section{Small scale protein expression}

The clones that have given a PCR product of the correct size were used to produce medium-scale cultures. Samples were taken every 24 hours for supernatant analysis. Culture medium was collected after 3 days culture in $250 \mathrm{ml}$ of YPD at $28^{\circ} \mathrm{C}$, by

Table 2 Primer sequences

\begin{tabular}{|c|c|c|}
\hline Name & Sequence & Mer \\
\hline a1AT-Forward & (5'-AAAGAATTCGAGGATCCCCAGGGAGATGC-3') & 29 \\
\hline a1AT-Reverse & (5'- TITTCTAGAGCTTATTITGGGTGGATTCACCAC-3') & 34 \\
\hline Ta1AT-Forward & (5'- AAAGAATTCAGCACCAATATCTTCTTCTC-3') & 29 \\
\hline Ta1AT-Reverse & (5'- TITTCTAGAGCTTATTTTGGGTGGATTCACCAC-3') & 34 \\
\hline pGAP -Forward & (5'-GTCCCTATTTCAATCAATTGAA-3') & 22 \\
\hline 3 AOX1 & (5'-GCAAATGGCATTCTGACATCC-3') & 21 \\
\hline
\end{tabular}


centrifugation at 4,000 g for $20 \mathrm{~min}$. The presence of recombinant proteins in the culture supernatant was determined by SDS PAGE, followed by Coomasie staining and Western Blot.

\section{Large scale protein expression}

To obtain large quantities of $\alpha 1 \mathrm{AT}$ fermentation was performed in a $5 \mathrm{~L}$ fermentor with a $4 \mathrm{~L}$ working volume YPD medium was initially used as the growth medium for $\alpha 1 \mathrm{AT}$ expression. To improve the growth characteristics of the cells in scaled-up fermentation, different media including YPD, YPG and YNB were examined, considering the salts and source of carbon. The rate of air supply and agitation was chosen as high as possible. Therefore, air was supplied at a rate of $10 \mathrm{l} / \mathrm{min}$ and the agitation rate was set at $400 \mathrm{rpm}$ under this scale.

\section{SDS-PAGE and silver staining}

The cells were removed and the supernatant was precipitated by using 100\% Aceton solution. After drying, the pellet was resuspended in loading buffer (10\% w/v SDS, $10 \mathrm{nM}$ $\beta$-mercaptoethanol, 20\% v/v Glycerol, $0.2 \mathrm{M}$ Tris-HCl, pH 6.8, 0.05\% Bromophenol blue w/v), heated for $10 \mathrm{~min}$, in boiling water and electrophoresed at 12\% SDSPAGE/100 V along with molecular weight marker (Fermentas). Gel was stained with silver nitrate according to Celis and coworkers [28].

\section{Western blot analysis}

Protein samples resolved on SDS-PAGE, were electro-blotted to Polyvinylidene Difluoride (PVDF) membrane (Millipore) in transferring buffer (0.025 M Tris, $0.19 \mathrm{M}$ glycine, and $20 \%(\mathrm{v} / \mathrm{v})$ methanol) overnight at $20 \mathrm{~V} / 4^{\circ} \mathrm{C}$. The membrane was treated with PBS-T-BSA (PBS, 0.1\% (v/v) Tween 20, 1\% (w/v) BSA) for 2 hours to block binding sites. After washing step, membrane was reacted with 1000 -fold diluted goat antihuman alpha-1 antitrypsin polyclonal antibody, conjugated with HRP (Abcam) for $3 \mathrm{hr}$. To eliminate non specific reactions, a supernatant of non- recombinant X-33 culture treated side by side accordingly as negative control. Subsequently, protein bands reacted positively, were visualized at the presence of 4-chloro1-naphtol substrate in PBS.

\section{Inhibitory activity assay}

Elastase activity was measured by EnzChek ${ }^{\ominus}$ Elastase Assay Kit (Molecular Probes, Inc.) according to the manufacturer's recommendations. The EnzChek kit contains $\mathrm{DQ}^{\mathrm{m}}$ elastin soluble bovine neck ligament elastin that has been labeled with BODIPY $^{\circ} \mathrm{FL}$ dye such that the conjugate's fluorescence is quenched. The nonfluorescent substrate can be digested by elastase or other proteases to yield highly fluorescent fragments. The presence of an inhibitor such as $\alpha 1 \mathrm{AT}$ blocks the substrate digestion hence subsequent fluorescent emission. The resulting change in fluorescence level was monitored using a standard fluorometer (Hitachi F-3010) with a maximum absorption at $505 \mathrm{~nm}$ and a maximum fluorescence emission at $515 \mathrm{~nm}$. Commercial human $\alpha 1 \mathrm{AT}$ was used as a positive control and the reaction buffer and the supernatant from non-recombinant $P$. pastoris (X-33 strain) culture as negative controls. 


\section{Results}

\section{Molecular dynamics simulation}

The root-mean-square deviation (RMSD)

One of the most frequently used measures of assessing the stability of an MD simulation over the course of time is the RMSD of the backbone atoms relative to the starting structure during the MD production phase. Although the RMSDs reach a stable value within the first ns, all the analyses have been carried out discarding the first three nanoseconds, i.e. over the last seven nanoseconds (Figure 1). This was done to guarantee an investigation over a well thermalized system.

\section{Gyration radius}

To determine the compactness of the chains during the simulations, the time-dependent squared radius of gyration, $\operatorname{Rg}^{2}(t)$ was determined. The radius of gyration gives an estimate of the characteristic volume of a globular polymer, which is inversely related to its compactness (Figure 2).

\section{Secondary structure comparison}

The analysis of the secondary structures, carried out with the program DSSP by sampling trajectories every 5 ps. Results indicates that the two proteins have comparable secondary structure regions. As shown in Table 3 no difference is observed in the structure of these $\alpha 1 \mathrm{AT}$ mutants (Table 3 ).

\section{Total energy changes}

Total energy changes during $10 \mathrm{~ns}$ of simulation time at the equilibrium phase under NPT conditions were carried out for the certitude of simulation stability (Figure 3 ). As
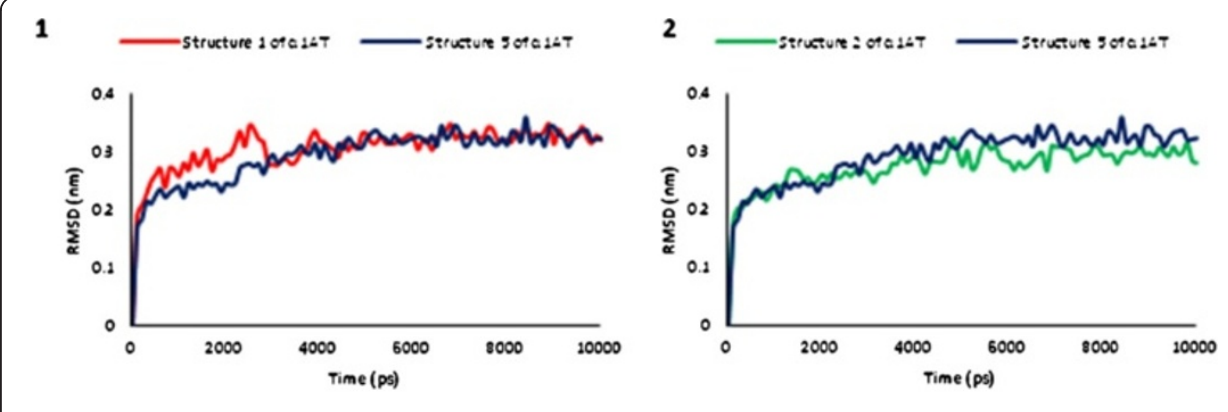

3
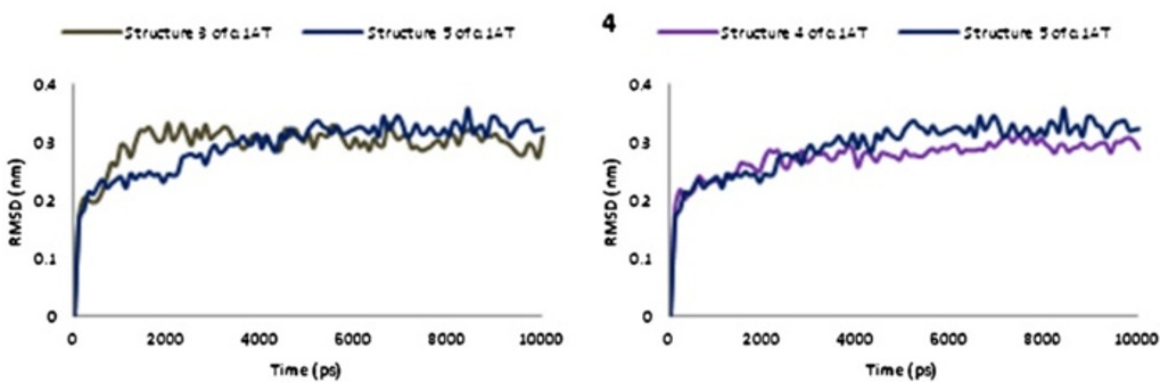

Figure 1 RMSD value of the backbone atoms of each a1AT structures in respect to the reference structure (a1AT5, blue) during $10 \mathrm{~ns}$ total simulation time. 


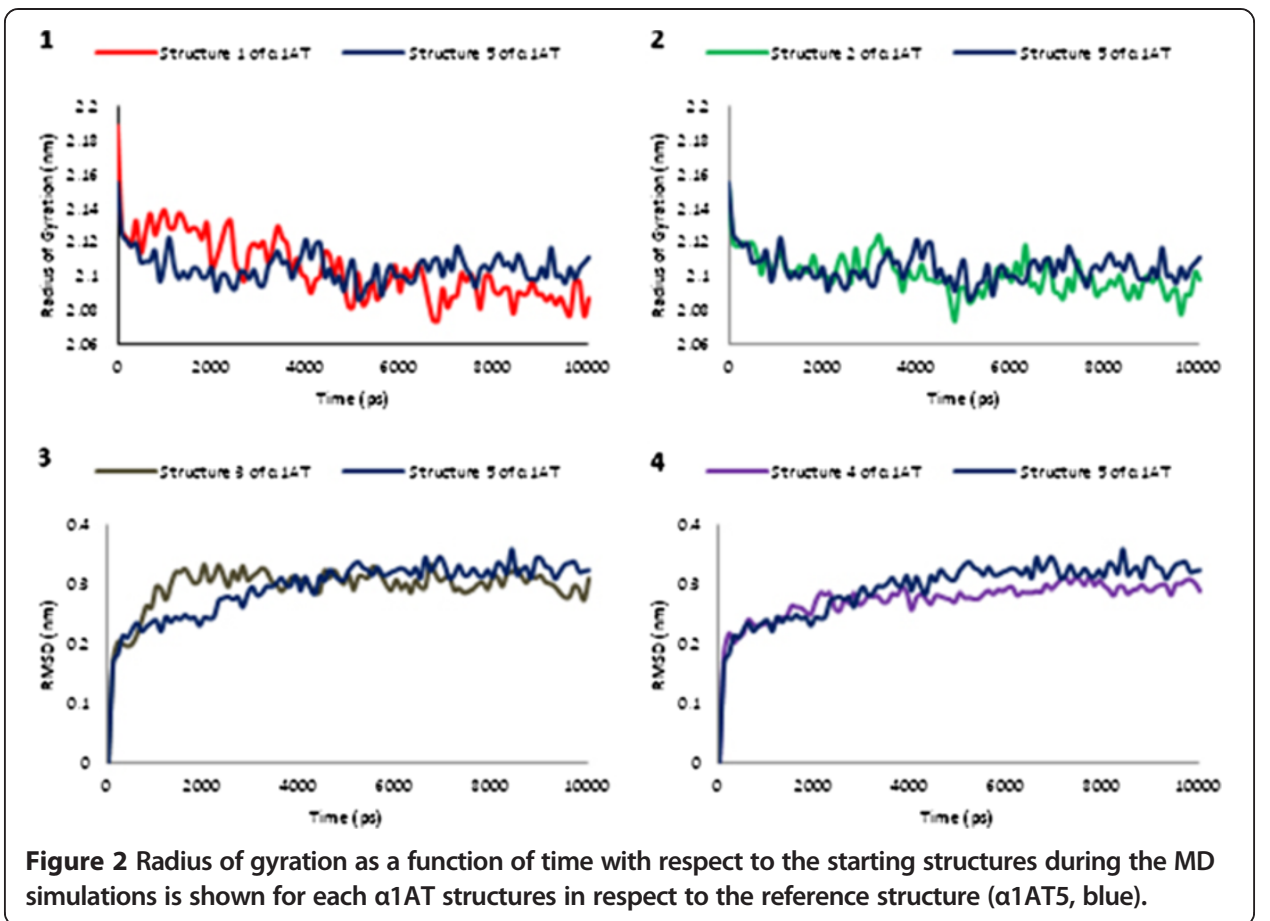

illustrated in Figure, the system's total energy at the equilibrium phase under fixed pressure conditions after 20 ps for all simulations remains constant.

\section{Temperature and density}

The temperature and the densities for all $\alpha 1 \mathrm{ATs}$ were $300 \mathrm{~K}$ and $1.00 \mathrm{gr} / \mathrm{cm}^{3}$, respectively that ultimately became fixed and stable, representing further proof of simulation stability (Figure 4).

\section{Structural fluctuations}

Figure 5 shows the $C^{\alpha}$ root mean square fluctuations (RMSF) of $\alpha 1 \mathrm{AT}$ structures in comparison to $\alpha 1 \mathrm{AT}$ structure 5 as a reference. The two $\alpha 1 \mathrm{AT}$ structures 2 and 4 show fewer fluctuations. As revealed in Figure 5, simulation of five structures indicate that a large part of residues is characterized by fluctuations not higher than $2.0 \AA$, apart from the regions of the $\mathrm{C}$ and $\mathrm{N}$-terminal tails. Therefore, the obtained structures from the simulation were optimal and the different structural regions affected each other. Hence, in each simulation, systems have reached to equilibrium.

Table 3 Secondary structure evolution of the five a1AT structures with simulation time

\begin{tabular}{lccccc}
\hline Secondary structure of $\mathbf{a 1 A T}$ & $\mathbf{1}$ & $\mathbf{3}$ & $\mathbf{5}$ & $\mathbf{2}$ & $\mathbf{4}$ \\
\hline $\boldsymbol{\beta}$ sheet + turn & 13.8328 & 13.8328 & 13.7837 & 13.7837 & 13.7837 \\
Coil & 72.6224 & 72.6224 & 72.7027 & 72.7027 & 72.7027 \\
$\boldsymbol{\beta}$ sheet & 12.1037 & 12.1037 & 12.1621 & 12.1621 & 12.1621 \\
Bend & 11.8155 & 11.8155 & 11.6216 & 11.6216 & 11.6216 \\
Turn & 1.7291 & 1.7291 & 1.6216 & 1.6216 & 1.6216 \\
3-Helix & 1.7291 & 1.7291 & 1.8918 & 1.8918 & 1.8918 \\
\hline
\end{tabular}



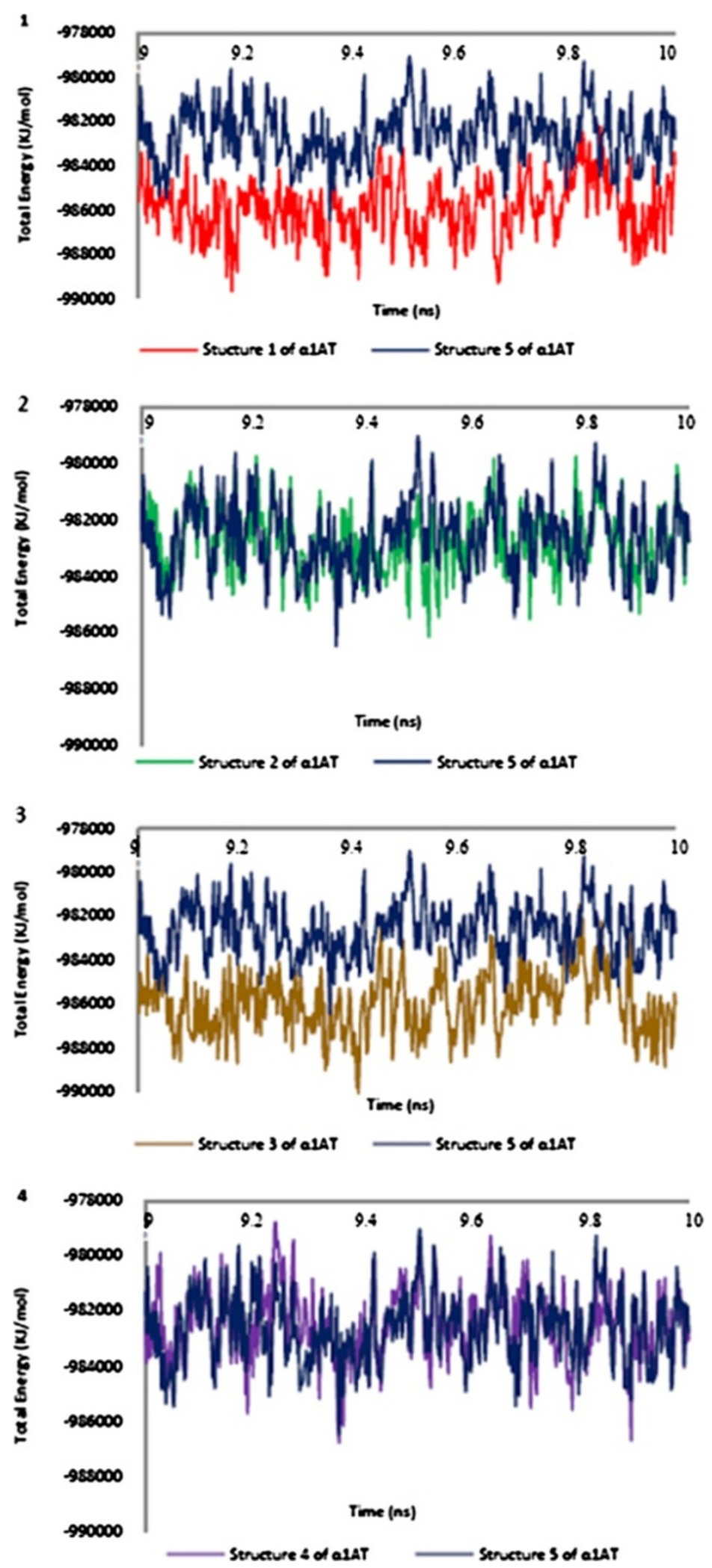

Figure 3 Plots of total energy versus time over the molecular dynamics trajectory for each a1AT structures in comparison to the reference structure (a1AT5, blue). 
A
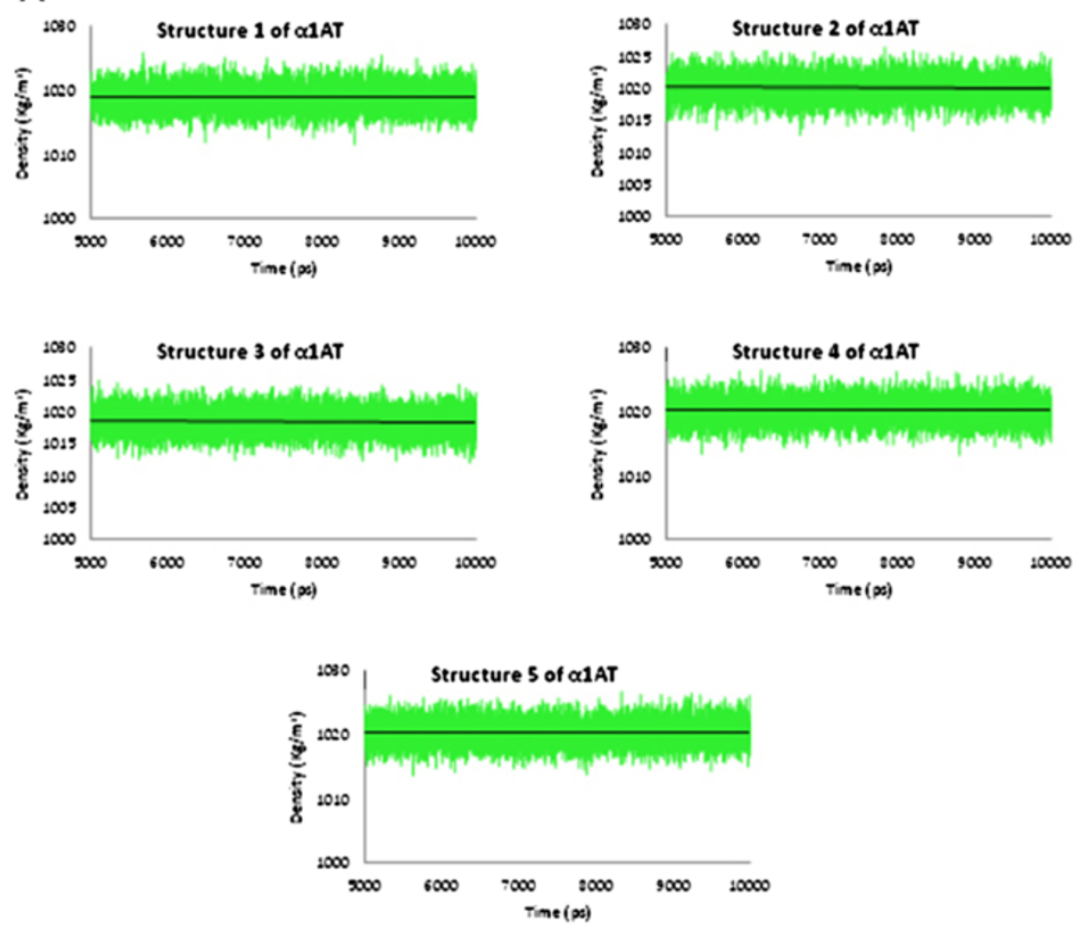

B
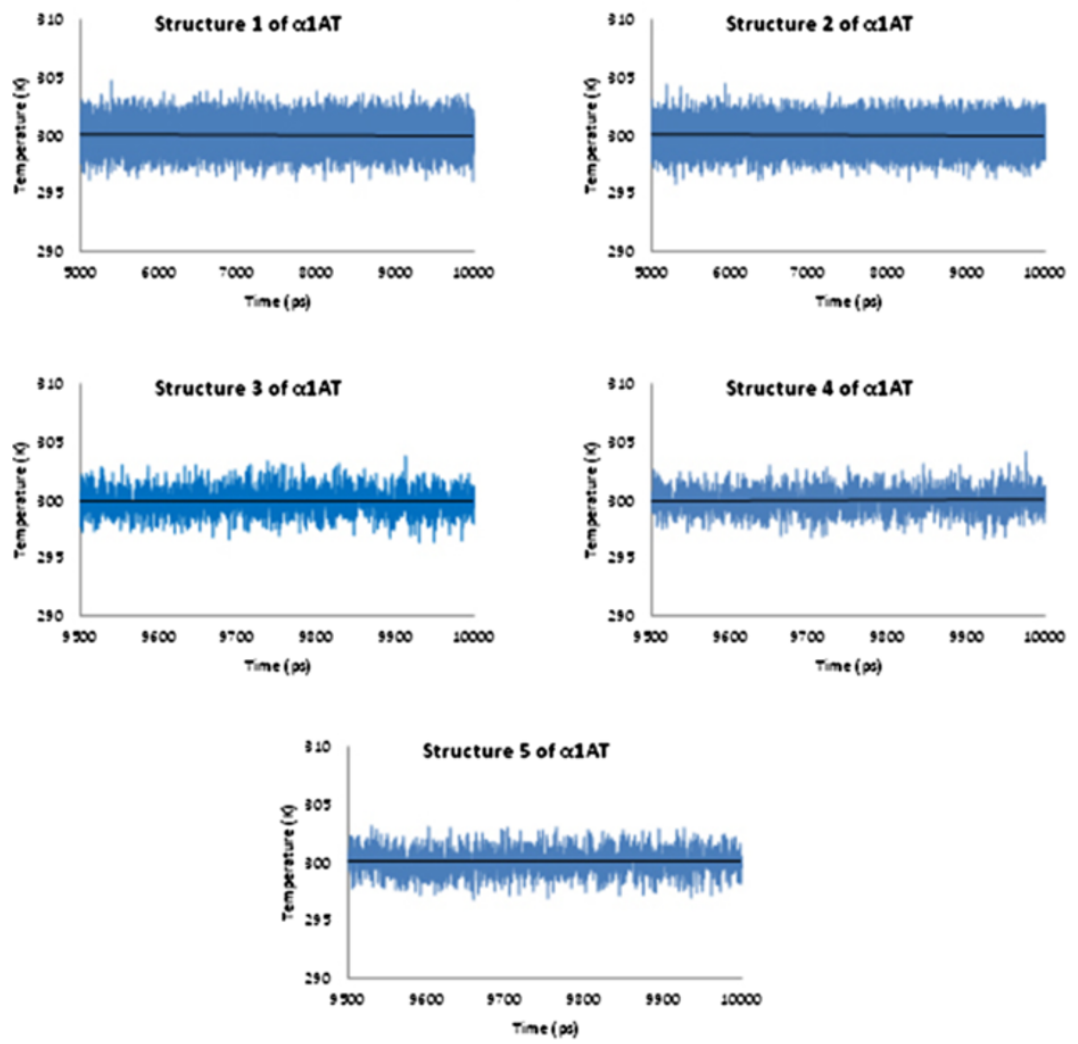

Figure 4 Plot of the system A) density and B) temperature during the 10 ns simulations of a1AT structures. 


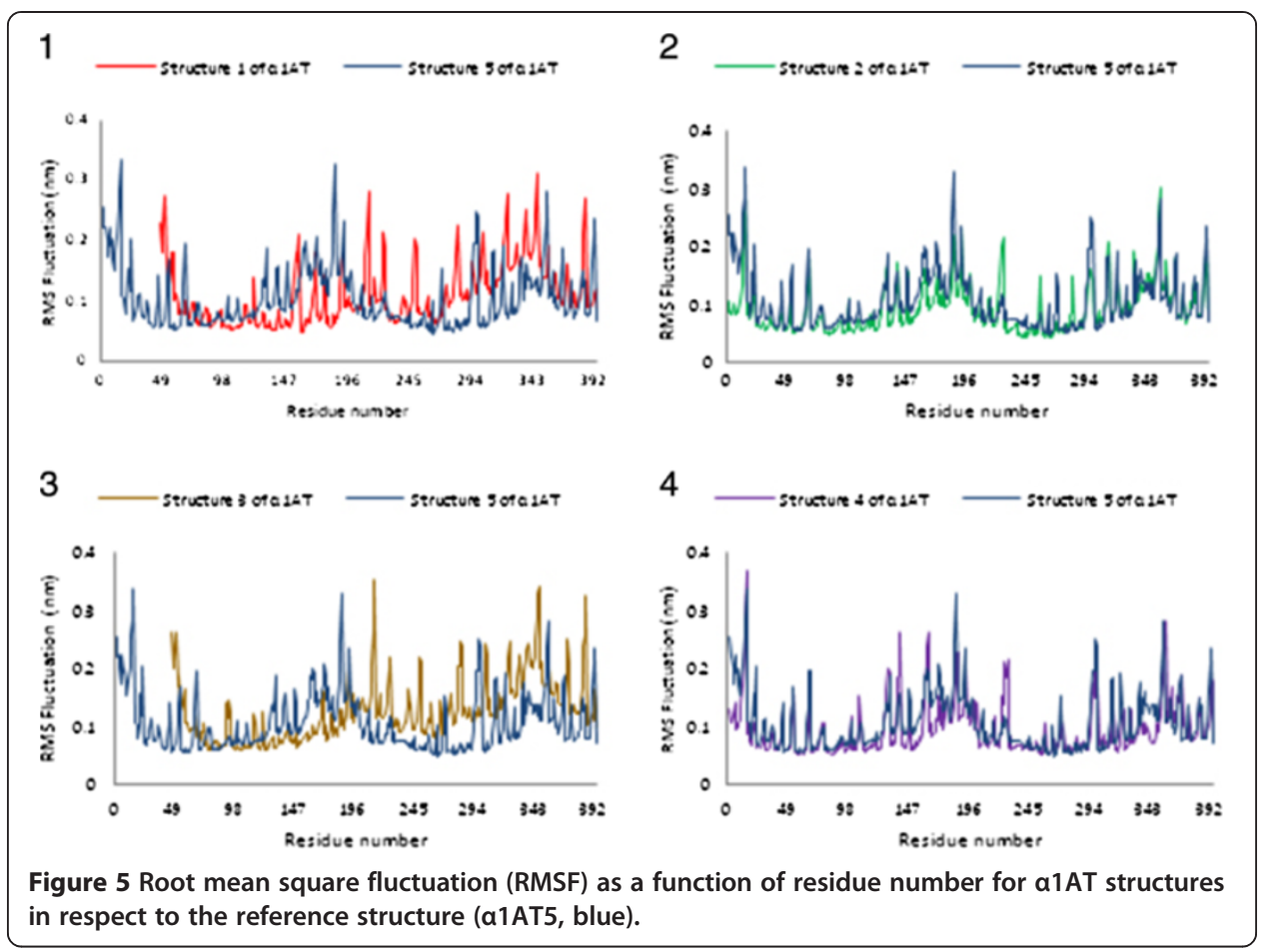

\section{Accessible surface area}

The accessible surface area (ASA) was computed considering contribution of side chain atoms and hydrophobic and hydrophilic contributions to the total ASA were calculated during the simulation time (Figure 6).

\section{Protein docking}

In each simulation 100 complexes were made, and complexes with the most binding affinity, which represent the best interaction in complexes, were selected (Table 4).

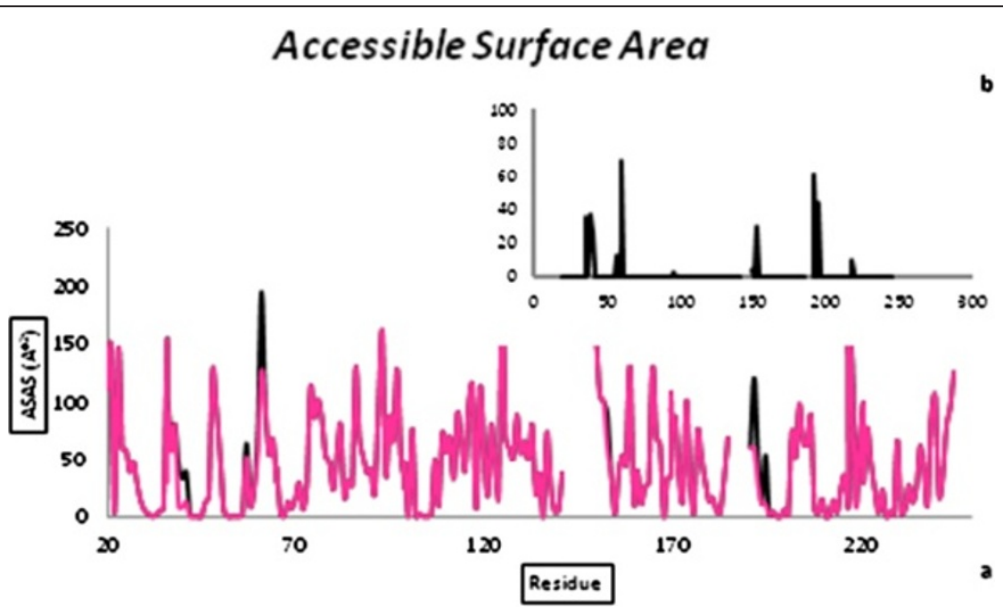

Figure 6 Comparison of accessible surface area of neutrophil elastase in the free and complex form. 
Table 4 Docking energy of a1AT structures and neutrophil elastase

\begin{tabular}{lc}
\hline Structure & Energy value \\
\hline a1AT1-Neutrophil Elastase Interaction & $-41.03 \mathrm{Kcal} / \mathrm{mol}$ \\
a1AT2-Neutrophil Elastase Interaction & $-46.42 \mathrm{Kcal} / \mathrm{mol}$ \\
a1AT3-Neutrophil Elastase Interaction & $-40.37 \mathrm{Kcal} / \mathrm{mol}$ \\
a1AT4-Neutrophil Elastase Interaction & $-41.93 \mathrm{Kcal} / \mathrm{mol}$ \\
a1AT5-Neutrophil Elastas Interaction & $-41.72 \mathrm{Kcal} / \mathrm{mol}$
\end{tabular}

Experimental studies

Integration analysis

Ten zeocin-resistant colonies of each $\alpha 1 \mathrm{AT}$ structures were selected. The integration of a1AT gene into the P. pastoris genome is verified by PCR. As shown in Figure 7, PCR products were separated on a $1 \%$ agarose gel and stained with ethidium bromide. All PCR fragments have the expected $\sim 1600 \mathrm{bp}$ sizes.

\section{SDS page}

The culture expressing native and truncated $\alpha 1 \mathrm{AT}$ was harvested at the following time intervals: $0,12,24,48,60,72$ and 96 hrs. The supernatant from each culture sample was examined using SDS-PAGE in order to determine the native and truncatedengineered $\alpha 1$ ATs expression levels. The $72 \mathrm{hrs}$ time period represented the best time for harvesting in comparison to non-recombinant X-33 culture (Figure 8).

\section{Western blot}

To confirm the identity of truncated-engineered proteins, proteins were reacted with $\alpha 1 \mathrm{AT}$ antibody in a western blot analysis. The recombinant $\alpha 1 \mathrm{AT}$ was identified as two distinct bands Western blotting using goat polyclonal to alpha-1 antitrypsin for native and truncated-engineered $\alpha 1$ ATs is shown in Figure 8, where the native and engineered proteins are detected in both Western blots.

\section{Elastase Inhibitory Capacity (EIC)}

The inhibitory function of native and truncated $\alpha 1 \mathrm{AT}$ showed no significant difference and both $\alpha 1$ ATs were found to be functional (Figure 9).

\section{Discussion}

Alpha 1- antitrypsin $(\alpha 1 \mathrm{AT})$ is a $54 \mathrm{kDa}$ glycoprotein which is a serine protease and broad group of other protease inhibitor. This glycoprotein contains 394 amino acids and three glycosylation sites. $\alpha 1 \mathrm{AT}$ protect lung from neutrophile elastase in inflammation or infection therefore it is called neutrophile elastase inhibitor [1,17]. The oxidation of Met358 in the RCL leads to a critical decrease in inhibitory capacity of this protein against elastase which cause inactivation in protective function of this protein. It was shown that oxidation sensitivity is a regulatory process, and $\alpha 1 \mathrm{AT}$ inactivation possibly causing lung tissue breakdown in inflammatory site through oxygen radicals released from phagocytes. Therefore, smoking causes oxidation of critical residues (methionine) in $\alpha 1 \mathrm{AT}$ and inactivates this protein $[14,15]$. $\alpha 1 \mathrm{AT}$ resistant to oxidation can improve health conditions in 

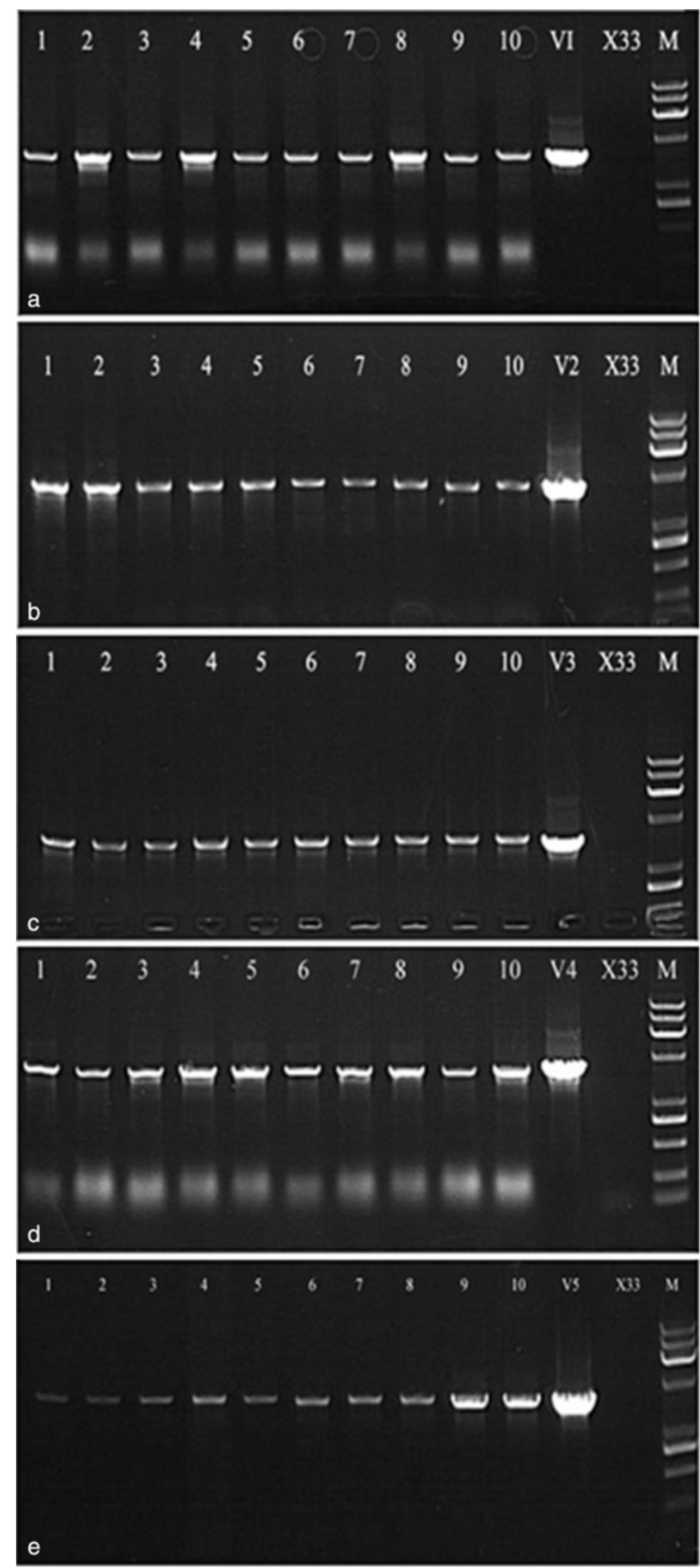

Figure 7 (See legend on next page.) 
(See figure on previous page.)

Figure 7 PCR colony screening was performed using plasmid-specific primers ( $p G A P$ forward and $\mathrm{Z}^{\prime} \mathrm{AOX}$ ) to determine positive clones. PCR screening of 10 Pichia pastoris strains transformed with pGAPZaA/a1AT plasmid. The presence of the specific a1AT -1600 bp PCR amplicon was detected by 1\% agarose gel electrophoresis (ethidium bromide staining), confirming the insertion of pGAPZ aA/a1AT plasmid to yeast genome. Lane 1-10: positive colonies of a1 $\mathrm{AT}_{1-5}$ constructs, V1-5: PCR positive control ( $p$ GAPZ aA plasmid) and X33: PCR negative control, M: molecular markers.
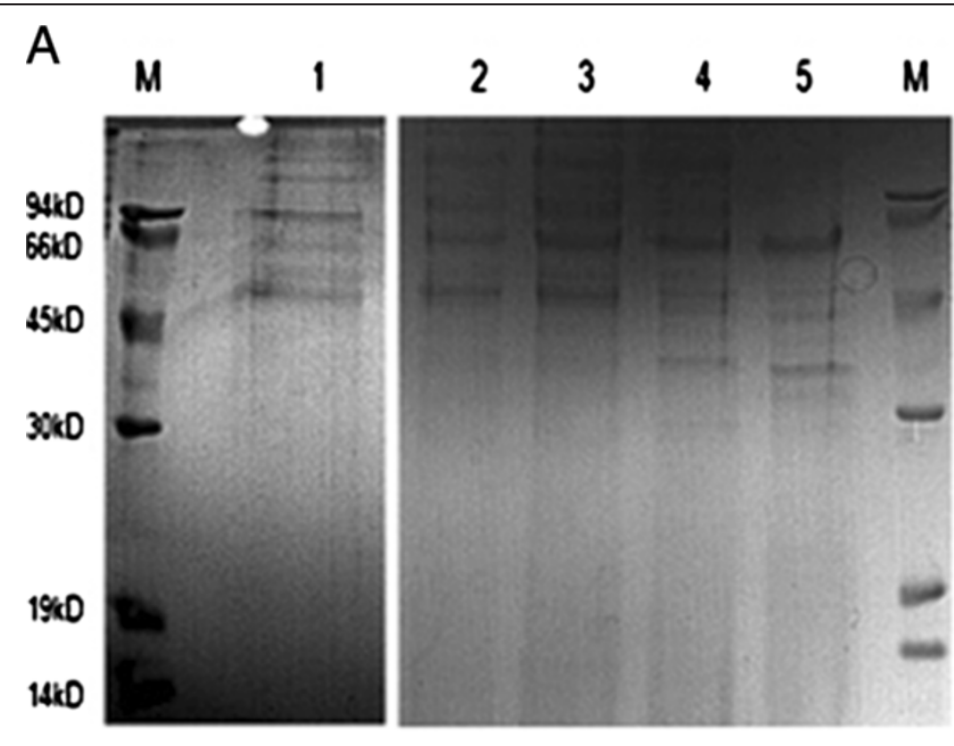

B

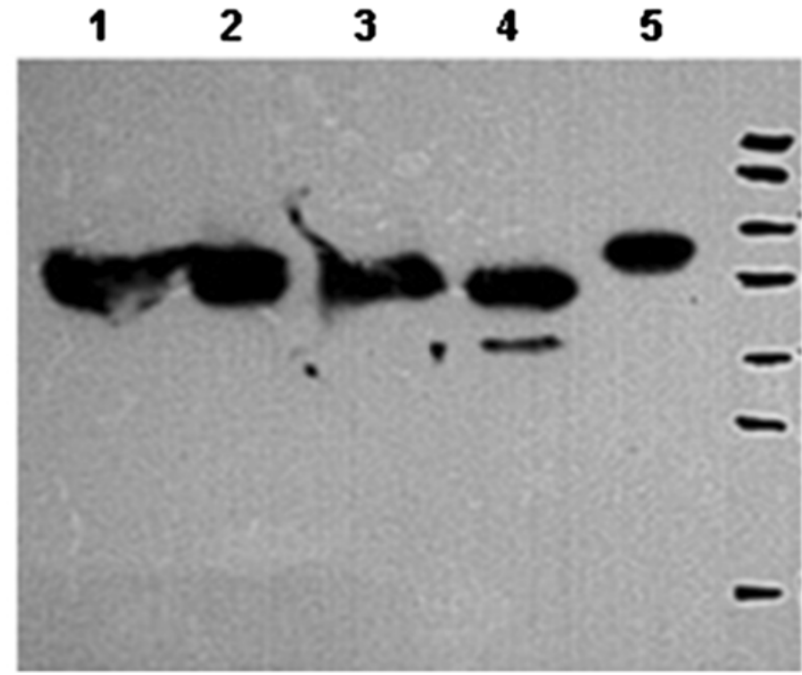

$95 \mathrm{kD}$ $72 \mathrm{kD}$ $55 \mathrm{kD}$ $43 \mathrm{kD}$ $34 \mathrm{kD}$ $26 \mathrm{kD}$

Figure 8 Expression analysis of constructed vectors containing different a1ATs sequences by SDSPAGE and western-blot. (A) $72 \mathrm{~h}$ after induction, supernatants were electrophoresed in $12 \%$ SDS-PAGE and stained by Coomassie blue as follows: lane $\mathrm{M}$, broad-range molecular weight marker; lanes 1, 2, 3, 4 and 5 supernatant samples of a1AT 1, 2, 3, 4 and 5, respectively. (B) Proteins were transferred onto a PVDF membrane and identified by immunoblotting using primary and secondary commercial antibodies as follows: lane $\mathrm{M}$, schematic representation of the broad-range marker; lane 1, 2, 3, 4 and 5, supernatant of $72 \mathrm{~h}$ inducing culture of a1 AT 1, 2, 3,4 and 5, respectively, which treated rabbit anti-AAT polyclonal antibody and then secondary Goat anti-rabbit polyclonal antibody. 


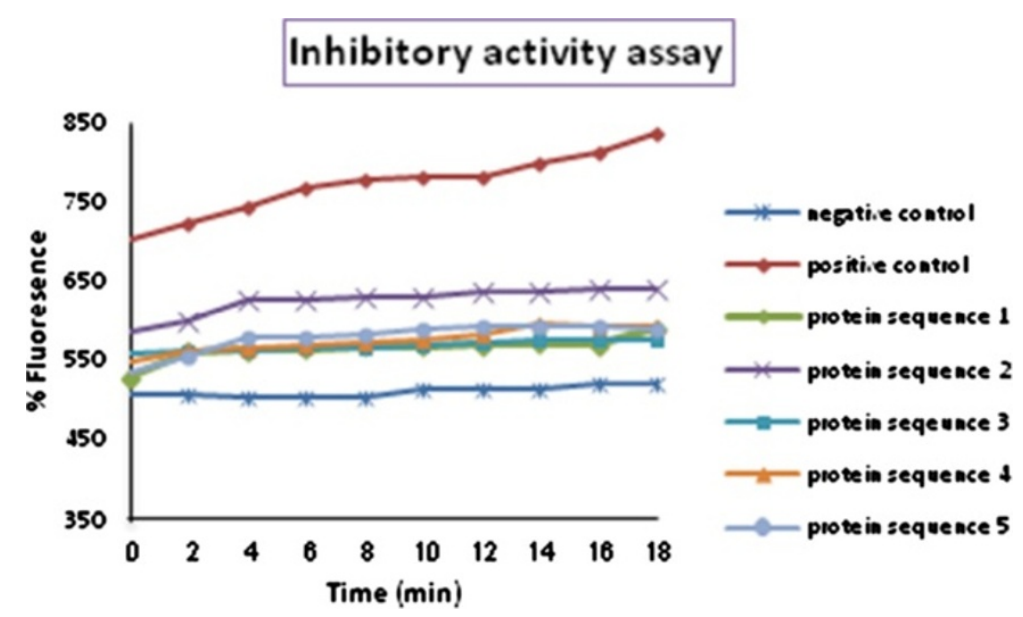

Figure 9 Elastase inhibitory assay of the five a1AT structures. a1AT and modified derivatives were incubated with a natural target of a1AT and monitored over $20 \mathrm{~min}$. The substrate is a BODIPY-FL-labeled DQ elastin conjugate that is highly labeled so that the fluorescence signal is quenched until enzymatic digestion yields highly fluorescent fragments. Since DQ elastin is digested by elastase, the inhibition of elastase activity by a1AT can be determined.

COPD patients and also reduction of apoptosis induced by cigarette smoking in vitro. The absence or inefficient function of $\alpha 1 \mathrm{AT}$ in the lungs leads to uncontrolled function of elastase and elastin breakdown, resulting in respiratory problems such as COPD and emphysema [3-5]. Association between $\alpha 1 \mathrm{AT}$ and a number of diseases including asthma, rheumatoid arthritis, anterior uveitis and systemic lupus erythematosus suggests that $\alpha 1 \mathrm{AT}$ is not only an anti-inflammatory protein but also an immune system regulator [8-10]. $\alpha 1 \mathrm{AT}$ regulates lymphocyte proliferation and cytotoxicity, mediates monocyte and neutrophil functions. Besides, researchers have shown that the protease-antiprotease imbalance is an important factor in the pathogenesis of COPD and other pulmonary diseases, such as bronchitis. COPD is one of the most important causes of irreversible lung damage and thus the fourth most common cause of death in the U.S. In this process, exogenous proteolytic enzymes lead to lung damage. Besides different physiological roles of $\alpha 1 \mathrm{AT}$ including the control of insulin secretion, antiprotease activity, protecting $\beta$-cells against cytokine-induced apoptosis, acting as an anti-inflammation compound [14-16], it is also regarded as an antiapoptotic factor in lung epithelial cells [11]. A recent study has shown that $\alpha 1 \mathrm{AT}$ can slow down the loss of insulin producing cells in diabetic patients who are at the early stages of disease. This study which is in phase II of clinical trials demonstrates the effect of $\alpha 1 \mathrm{AT}$ in preservation of $\beta$-cells' function $[18,19]$. Therefore, only with appropriate and adequate concentrations of $\alpha 1 \mathrm{AT}$ the lungs' correct function can be maintained.

One of the treatment strategies for optimum function of $\alpha 1 \mathrm{AT}$ in inflammation is the replacement therapy using intravenous infusion. In the infusion form only $10 \%-15 \%$ of $\alpha 1 \mathrm{AT}$ reach the target organ. Another possible treatment strategy is by airway delivery. In this form of treatment not only the aerosolized $\alpha 1 \mathrm{AT}$ reach directly the target organ, but also prevent accumulation of excess drug in blood and therefore much lower amount of drug is require $[18,29]$. Furthermore, in addition to the development and 
optimization of recombinant form of $\alpha 1 \mathrm{AT}$, efforts on packaging $\alpha 1 \mathrm{AT}$ in micro and nanoparticles for pulmonary delivery are also under investigations.

During drug delivery, especially protein-based ones, the size of the protein has the major role with regard to its packaging and delivery. In other words, the smaller the protein size, its packaging, stability and delivery becomes more convenient. Protein length from $\mathrm{N}$-terminal was reduced to produce a smaller molecule which has the same and even higher inhibitory activity as the native $\alpha 1 \mathrm{AT}$. Previous studies have shown that the two methionine residues 351 and 358, and in sever conditions methionine 226 are susceptible to oxidation. Oxidation of methionine 358 leads the loss of anti-elastase activity of $\alpha 1 \mathrm{AT}$. The other methionine residue (Met351) is also as susceptible to oxidation and anti-elastase activity loss as Met358 [23,24].

Therefore, five different $\alpha 1 \mathrm{AT}$ structures with different glycosylation patterns and molecular weights were constructed base of theoretical studies. Beside, each $\alpha 1$ AT structures were subjected to three or five different site directed mutagenesis at the same time for both thermal and oxidative inactivation stability. Inhibitory properties of each $\alpha 1 \mathrm{AT}$ constructs were investigated through Elastase Inhibitory Concentration (EIC).

In this study, site directed mutations in $\alpha 1 \mathrm{AT}$, produce an active, lower molecular weight, resistant to oxidation and thermal inactivation, with an appropriate half life and unglycosylated protein in Pichia Pastoris as a host. In this study we showed that mutations and engineering of the protein molecule, lead to an improvement in inhibitory function. Furthermore, a protein with a less molecular weight and immunogenicity which is suitable for drug packaging and targeting to the lung which is the site of a1AT's action was produce. Molecular dynamic studies showed that deletion of the first alpha-helix from $\mathrm{N}$-terminal sequence have no effect on $\alpha 1 \mathrm{AT}$ inhibitory function. Therefore a reduction of $7 \mathrm{kDa}$ in molecular weight of $\alpha 1 \mathrm{AT}, 5 \mathrm{kDa}$ contributing to amino acids weight and approximating $2 \mathrm{kDa}$ to the first carbohydrate chain, was resulted.

Before entering the experimental phase of studies, the effect of eliminating the first 46 amino acids from the N-terminal region, on protein structure was investigated using MD simulation. The native and truncated structures were modeled and the results of the theoretical studies showed no meaningful differences between the properties of these proteins. Results from the dynamic simulations of the molecular models and their interactions with neutrophil elastase showed different interaction energy. The docking energy for the $\alpha 1$ AT 2 is less negative which shows better interaction with neutrophil elastase. These findings provided the basis for the experimental phase of the study in which sequences from the five $\alpha 1 \mathrm{AT}$ constructs were inserted into the expression vector pGAPZ $\alpha . \alpha 1$ ATs were expressed in the yeast Pichia pastoris (P. pastoris) in a secretary manner and under GAP promoter transformed into Pichia pastoris. Thus, experimental studies were carried out to produce recombinant proteins. The level of expression and the functions of the native and truncated proteins were compared. Protein inhibitory activity was investigated through EIC (elastase inhibitory capacity) Results show that all five constructs have a good elastase inhibitory function, although, $\alpha 1$ AT 2 has the highest inhibitory activity even more than the native $\alpha 1 \mathrm{AT}$.

In conclusion, a recombinant and truncated protein with oxidative resistance and without the two main glycosylation chains were obtained which do not effect the inhibitory activity of the $\alpha 1 \mathrm{AT} 2$. The results have important influence in pulmonary drug delivery. 


\section{Competing interests}

The authors declare that they have no competing interests.

\section{Authors' contributions}

NP wrote this manuscript and carried out most of the experiments. SH was main supervisor of this research in Tarbiat Modares University and National Institute of Genetic Engineering and Biotechnology. SSA provided guidance and assistance in modelling and simulation. Professor ASL from Tarbiat Modares University and National Institute of Genetic Engineering and Biotechnology advised on experimental part. Professor MG from Baqiyatallah University of Medical Sciences contributed gave us valuable guidance to improve this work. AS provided assistance in the data analysis. All authors read and approved the final manuscript.

\section{Acknowledgments}

The authors thank Mr. Saman Ashtiani, for his assistance in molecular dynamics simulation. Part of the computational work was carried out at the Modeling Laboratory of Tarbiat Modares University.

\section{Author details}

${ }^{1}$ Department of Biology, Faculty of Sciences, University of Guilan, Rasht, Iran. ${ }^{2}$ Department of Biochemistry, School of Biological Sciences, Tarbiat Modares University, Tehran, Iran. ${ }^{3}$ National Institute of Genetic Engineering and Biotechnology, Tehran, Iran. ${ }^{4}$ Department of Biophysics, School of Biological Sciences, Tarbiat Modares University, Tehran, Iran. ${ }^{5}$ Department of Clinical Biochemistry, Faculty of Medical Sciences, Tarbiat Modares University, Tehran, Iran.

${ }^{6}$ Research Center for Chemical Injuries, Baqiyatallah University of Medical Sciences, Tehran, Iran.

Received: 23 March 2013 Accepted: 13 May 2013

Published: 24 May 2013

\section{References}

1. Kalsheker N: Alpha 1-antitrypsin: structure, function and molecular biology of the gene. Biosci Rep 1989, 9(2):129-138.

2. Schlade-Bartusiak K, Cox DW: Alpha1-antitrypsin deficiency. In Gene Reviews [Internet]. Seattle (WA). Edited by Pagon RA, Bird TD, Dolan CR, Stephens K, Adam MP. Seattle: University of Washington; 1993.

3. Stoller JK, Aboussouan LS: Alpha1-antitrypsin deficiency. Lancet 2005, 365(9478):2225-2236.

4. Crystal RG: Alpha 1-antitrypsin deficiency, emphysema, and liver disease. Genetic basis and strategies for therapy. J Clin Invest 1990, 85(5):1343-1352.

5. Furey NL, Golden RS, Potts SR: Treatment of alpha-1-antitrypsin deficiency, massive edema, and panniculitis with alpha-1 protease inhibitor. Ann Intern Med 1996, 125(8):699.

6. O'Riordan K, Blei A, Rao MS, Abecassis M: alpha 1-antitrypsin deficiency-associated panniculitis: resolution with intravenous alpha 1-antitrypsin administration and liver transplantation. Transplantation 1997, 63(3):480-482.

7. Blanco I, Lara B, de Serres F: Efficacy of alpha1-antitrypsin augmentation therapy in conditions other than pulmonary emphysema. Orphanet J Rare Dis 2011, 6:14.

8. Dowd SK, Rodgers GC, Callen JP: Effective treatment with alpha 1-protease inhibitor of chronic cutaneous vasculitis associated with alpha 1-antitrypsin deficiency. J Am Acad Dermatol 1995, 33(5 Pt 2):913-916.

9. Blanco I, Janciauskiene S, Nita I, Fernandez-Bustillo E, Carcaba V, Gallo C, Alvarez-Rico M, de Serres F, Beridze N: Low plasma levels of monocyte chemoattractant protein-1 (MCP-1), tumor necrosis factor-alpha (TNFalpha), and vascular endothelial growth factor (VEGF) in patients with alpha1-antitrypsin deficiency-related fibromyalgia. Clin Rheumatol 2010, 29(2):189-197.

10. Tonelli AR, Brantly ML: Augmentation therapy in alpha-1 antitrypsin deficiency: advances and controversies. Ther Adv Respir Dis 2010, 4(5):289-312.

11. Sivasothy P, Dafforn TR, Gettins PG, Lomas DA: Pathogenic alpha 1-antitrypsin polymers are formed by reactive loop-beta-sheet A linkage. J Biol Chem 2000, 275(43):33663-33668.

12. Gotzsche $P C$, Johansen HK: Intravenous alpha-1 antitrypsin augmentation therapy for treating patients with alpha-1 antitrypsin deficiency and lung disease. Cochrane Database Syst Rev 2010, 7, CD007851.

13. Rubin BK: Therapeutic aerosols and airway secretions. J Aerosol Med 1996, 9(1):123-130.

14. Hopkins PC, Carrell RW, Stone SR: Effects of mutations in the hinge region of serpins. Biochemistry 1993, 32(30):7650-7657.

15. Irving JA, Pike RN, Lesk AM, Whisstock JC: Phylogeny of the serpin superfamily: implications of patterns of amino acid conservation for structure and function. Genome Res 2000, 10(12):1845-1864.

16. Crystal RG, Brantly ML, Hubbard RC, Curiel DT, States DJ, Holmes MD: The alpha 1-antitrypsin gene and its mutations. Clinical consequences and strategies for therapy. Chest 1989, 95(1):196-208.

17. Karnaukhova E, Ophir Y, Golding B: Recombinant human alpha-1 proteinase inhibitor: towards therapeutic use. Amino Acids 2006, 30(4):317-332.

18. Courrier HM, Butz N, Vandamme TF: Pulmonary drug delivery systems: recent developments and prospects. Crit Rev Ther Drug Carrier Syst 2002, 19(4-5):425-498.

19. Casolaro MA, Fells G, Wewers M, Pierce JE, Ogushi F, Hubbard R, Sellers S, Forstrom J, Lyons D, Kawasaki G, et al: Augmentation of lung antineutrophil elastase capacity with recombinant human alpha-1-antitrypsin. J Appl Physiol 1987, 63(5):2015-2023.

20. Cregg JM, Cereghino JL, Shi J, Higgins DR: Recombinant protein expression in Pichia pastoris. Mol Biotechnol 2000, 16(1):23-52.

21. Gerngross TU: Advances in the production of human therapeutic proteins in yeasts and filamentous fungi. Nat Biotechnol 2004, 22(11):1409-1414.

22. Pemberton PA, Bird PI: Production of serpins using yeast expression systems. Methods 2004, 32(2):185-190. 
23. Taggart C, Cervantes-Laurean D, Kim G, McElvaney NG, Wehr N, Moss J, Levine RL: Oxidation of either methionine 351 or methionine 358 in alpha 1-antitrypsin causes loss of anti-neutrophil elastase activity. J Biol Chem 2000, 275(35):27258-27265.

24. Brantly M: Alpha1-antitrypsin: not just an antiprotease: extending the half-life of a natural anti-inflammatory molecule by conjugation with polyethylene glycol. Am J Respir Cell Mol Biol 2002, 27(6):652-654.

25. Zhang Y: I-TASSER server for protein 3D structure prediction. BMC Bioinformatics 2008, 9:40.

26. Hess B, Kutzner C, van der Spoel D, Lindahl E: GROMACS 4: algorithms for highly efficient, load-balanced, and scalable molecular simulation. J Chem Theory Comput 2008, 4(3):435-447.

27. Berendsen HJC, van der Spoel D, van Drunen R: GROMACS: a message-passing parallel molecular dynamics implementation. Comp Phys Comm 1995, 91:43-56.

28. Celis JE, Carter N, Hunter T, Shotton D, Simons K, Small JV: Cell biology: A Laboratory Handbook, vol. 4, third edn. San Diego, CA: Academic; 1998.

29. Hubbard RC, Crystal RG: Strategies for aerosol therapy of alpha 1-antitrypsin deficiency by the aerosol route. Lung 1990, 168(Suppl):565-578.

doi:10.1186/1742-4682-10-36

Cite this article as: Pirooznia et al: The design of a new truncated and engineered alpha1-antitrypsin based on theoretical studies: an antiprotease therapeutics for pulmonary diseases. Theoretical Biology and Medical Modelling 2013 10:36

\section{Submit your next manuscript to BioMed Central and take full advantage of:}

- Convenient online submission

- Thorough peer review

- No space constraints or color figure charges

- Immediate publication on acceptance

- Inclusion in PubMed, CAS, Scopus and Google Scholar

- Research which is freely available for redistribution

Submit your manuscript at www.biomedcentral.com/submit 